\title{
Fatores de risco associados ao transtorno depressivo maior em uma comunidade
}

\section{universitária}

\author{
Association between risk factors and major depressive disorder in a university community \\ Factores de riesgo asociados al trastorno depresivo mayor en una comunidad universitária
}

Recebido: 22/11/2021 | Revisado: 02/12/2021 | Aceito: 03/12/2021 | Publicado: 05/12/2021

\author{
Thayná Moraes Ferreira \\ ORCID: https://orcid.org/0000-0002-1560-8807 \\ Universidade Federal do Pará, Brasil \\ E-mail: thaynamf@gmail.com \\ Claudia Ximena Bobadilla Chavez \\ ORCID: https://orcid.org/0000-0002-3901-5424 \\ Universidade Federal do Pará, Brasil \\ E-mail: claudiabch2705@gmail.com \\ José Ramon Gama Almeida \\ ORCID: https://orcid.org/0000-0002-9871-0531 \\ Universidade Federal do Pará, Brasil \\ E-mail: ramonalmeida1703@gmail.com \\ Rayan Fidel Martins Monteiro \\ ORCID: https://orcid.org/0000-0001-6642-4488 \\ Universidade Federal do Pará, Brasil \\ E-mail: rayanfidel@hotmail.com \\ Paulo Eduardo Santos Avila \\ ORCID: https://orcid.org/0000-0002-4806-3719 \\ Universidade Federal do Pará, Brasil \\ E-mail: pauloavila@yahoo.com \\ Gilmara de Nazareth Tavares Bastos \\ ORCID: https://orcid.org/0000-0002-4899-6500 \\ Universidade Federal do Pará, Brasil \\ E-mail: bastosgnt @gmail.com
}

\begin{abstract}
Resumo
Objetivo: Foi realizada uma análise descritiva do perfil sociodemográfico dos pacientes diagnosticados com transtorno depressivo maior; além de estabelecer uma correlação sobre fatores pessoais, parâmetros de sensibilidade somática, características fisiológicas e uso de tratamento farmacológico do grupo com TDM e indivíduos saudáveis. Métodos: Foi analisado o banco de dados no período de março a maio de 2019, referente as fichas de avaliação aplicadas aos funcionários e discentes da Universidade Federal do Pará, posteriormente foi feita a avaliação estatística por meio do programa Microsoft Office Excel® 2013 e do Bioestat - versão 5.3. Resultados: No perfil sociodemográfico dos 50 participantes avaliados, $29(58 \%)$ pertenciam ao sexo feminino, com uma forte associação entre o sexo $(p=0.0219)$ com esse transtorno mental. $O$ funcionamento intestinal alterado apresentou um aumento de $100 \%$ entre o GTDM e o GC. Dentre as variáveis referentes as sensações somáticas, a presença do prurido foi a variável com importante diferença entre os grupos, sendo o GC 23 (92\%) sem alteração de sensibilidade a coceira, em comparação ao GTDM 11 (44\%) pacientes relataram a presença do prurido. Houve associação estatisticamente forte entre a queda de cabelo e a qualidade do sono no grupo com transtorno depressivo. Conclusão: Conclui-se que os pacientes com TDM apresentam fatores de risco próprios do indivíduo e sintomas somáticos que influenciam no curso de resolução da doença. Sugere-se a realização de mais estudos para relacionar características sociodemográficas, presença de sintomas somáticos e interação medicamentosa para identificar possíveis fatores associados ao quadro de depressão maior na população universitária.
\end{abstract}

Palavras-chave: Transtorno depressivo maior; Sintoma somático; Fatores de risco; Serviços de saúde na universidade; Saúde pública.

\footnotetext{
Abstract

Objective: A descriptive analysis of the sociodemographic profile of patients diagnosed with major depressive disorder was performed; in addition to establishing a correlation on personal factors, somatic sensitivity parameters, physiological characteristics and use of pharmacological treatment in the group with MDD and healthy individuals. Methods: The database was analyzed from March to May 2019, referring to the evaluation sheets applied to employees and students of the Federal University of Pará, later the statistical evaluation was carried out using the Microsoft Office Excel ${ }^{\circledR} 2013$ program and the Bioestat - version 5.3. Results: In the sociodemographic profile of the 50 participants evaluated, the majority were female $29(58 \%)$, with a strong association between gender $(\mathrm{p}=0.0219)$
} 
with this mental disorder. Altered regular bowel function showed a 100\% increase between GTDM and GC. Among the variables related to somatic sensations, the presence of pruritus was the variable with an important difference between the groups, with CG 23 (92\%) without change in sensitivity to itching, compared to GTDM 11 (44\%) patients reported the presence of pruritus. There was a statistically strong association between hair loss and sleep quality in the depressive disorder group. Conclusion: It is concluded that patients with major depressive disorder have risk factors specific to the individual and somatic symptoms that influence the course of disease resolution. Further studies are suggested to relate sociodemographic characteristics, presence of somatic symptoms and drug interactions to identify possible factors associated with major depression in the university population.

Keywords: Major depressive disorder; Somatic symptom; Risk factors; Student health services; Public health.

\section{Resumen}

Objetivo: Análisis descriptivo del perfil sociodemográfico de pacientes diagnosticados de trastorno depresivo mayor; además de establecer una correlación sobre factores personales, parámetros de sensibilidad somática, características fisiológicas y uso de tratamiento farmacológico en el grupo con TDM e individuos sanos. Métodos: La base de datos se analizó de marzo a mayo de 2019, refiriéndose a los formularios de evaluación aplicados a empleados y estudiantes de la Universidad Federal de Pará, posteriormente se realizó la evaluación estadística utilizando el programa Microsoft Office Excel® 2013 y el Bioestat - versión 5.3. Resultados: En el perfil sociodemográfico de los 50 participantes evaluados, $29(58 \%)$ eran mujeres, con una fuerte asociación de género $(\mathrm{p}=0,0219)$ con este trastorno mental. La función intestinal alterada mostró un aumento del 100\% entre GTDM y GC. Entre las variables relacionadas con las sensaciones somáticas, la presencia de prurito fue la variable con una diferencia importante entre los grupos, con CG 23 (92\%) sin cambio en la sensibilidad al prurito, en comparación con GTDM 11 (44\%) los pacientes reportaron la presencia de prurito. Hubo una asociación estadísticamente fuerte entre la pérdida de cabello y la calidad del sueño en el GTDM. Conclusión: Se concluye que los pacientes con TDM tienen factores de riesgo específicos del individuo y síntomas somáticos que influyen en el curso de la resolución de la enfermedad. Se sugieren más estudios que relacionen características sociodemográficas, presencia de síntomas somáticos e interacciones farmacológicas para identificar posibles factores asociados a la depresión mayor en la población universitaria.

Palabras clave: Trastorno depresivo mayor; Síntoma somático; Factores de riesgo; Servicios de salud para estudiantes; Salud pública.

\section{Introdução}

A depressão é um transtorno mental altamente prevalente em todo o mundo, que influencia nos processos fisiológicos, sociais e psicológicos que constituem o ser humano e a vida em comunidade, tornando-se um problema de saúde pública universal até 2030, com efeitos críticos na qualidade de vida individual e social (Athira, K. et al., 2020).

Dados da Organização Mundial da Saúde (2018) revelam que a depressão é a principal causa de deficiência global, mais de 300 milhões de pessoas no mundo são afetadas por esta condição, no qual 5\% dessa população é formada por adultos, sendo as mulheres diagnosticadas duas vezes mais em comparação aos homens (Otte C, et al., 2016; World Health Organization -HWO, 2018).

No Brasil, aproximadamente 11,5 milhões de pessoas apresentam depressão (Gonçalves, A. M. C., et al., 2017; Medeiros, S. P., et al., 2020). De modo que uma parcela dessa população, aproximadamente 15 a $25 \%$, é encontrada no ambiente acadêmico, no qual as demandas psicossociais tendem a ser maximizadas e comprometem o desempenho e a qualidade de vida, tornando esse grupo social mais vulnerável e propenso a desenvolver transtornos mentais, destacando-se a depressão (Santos, K., et al., 2018).

Durante a universidade os acadêmicos passam por grandes mudanças na rotina que os tornam responsáveis por cumprir uma série de obrigações, conciliar adversidades e cobranças de desempenho. Tais situações podem intensificar algum problema de saúde mental pré-existente ou ser um fator contribuinte para que um novo transtorno psiquiátrico venha à tona (Fernández-Rodríguez C., et al., 2019).

O cotidiano de servidores e docentes universitários é permeado por fatores agravantes que tornam esse público suscetível para o desenvolvimento de transtornos mentais (Prado, CEP, 2016; Silva \& Tucci, 2018). As exigências da carreira acadêmica, a carga horária extensa e a negligência com o lazer e o descanso contribuem para elevar os níveis de estresse laboral e, assim, 
gerar uma resposta inespecífica do corpo diante das demandas impostas que evoluem de forma negativa para a saúde do trabalhador (Prado, CEP, 2016; Silva \& Tucci, 2018).

O transtorno depressivo maior (TDM) é um transtorno psiquiátrico debilitante que atinge a população em geral em um amplo espectro de idades e origens sociais, caracterizado por humor deprimido, diminuição de interesses, comprometimento da função cognitiva e sintomas vegetativos, como distúrbios do sono ou apetite, que são acompanhados de alterações somáticas que afetam significativamente a capacidade de funcionamento do indivíduo (American Psychiatric Association, 2014).

A etiologia e a fisiopatologia do TDM permanecem amplamente desconhecidas, as principais vias discutidas incluem alterações fisiopatológicas em sistemas heterogêneos, como sistemas genéticos, epigenéticos, funções de neurotransmissores e neuromoduladores, fatores neurotróficos, eixo hipotálamo-hipófise-adrenal, sistema imunológico, bem como mecanismos de estresse celular (Pitsillou, E., et al., 2019). Ademais, a exposição a circunstâncias estressantes da vida é um dos fatores de risco mais fortes para o desenvolvimento de depressão, em decorrência do desenvolvimento de respostas inflamatórias no cérebro e na periferia (Stetler, C., \& Miller, G. E. 2011).

Esses componentes interagem entre si gerando uma matriz complexa resultando em mecanismos patogênicos considerados perturbadores da homeostase interna do corpo. Quando ocorrem eventos estressores repetidos, o indivíduo é afetado pelo acúmulo de demandas psicológicas e físicas que podem ocasionar uma doença com um curso pleomórfico, com variações consideráveis na remissão e cronicidade.

Em pesquisas baseadas na população, aproximadamente 70-90\% dos pacientes com TDM se recuperam dentro de 1 ano. No entanto, a chance de recorrência de TDM é alta, pois cerca de $80 \%$ dos pacientes em remissão experimentam pelo menos uma recorrência em toda a vida. De maneira que após a interrupção do TDM, os sintomas residuais, a capacidade funcional e a percepção sensorial permanecem, alterando o bem-estar global do indivíduo. (Otte C, et al., 2016).

O sistema somatossensorial nos permite apreciar algumas das experiências mais agradáveis, como também algumas das mais desagradáveis. A sensação somática permite que o nosso corpo sinta o contato, a dor, o frio e que saiba reconhecer quais partes dele estão sendo estimuladas (Pereira, 2014). Nessa conformidade, os transtornos psiquiátricos apresentam sintomas somáticos caracterizados por serem queixas corporais recorrentes, que não possuem causas estruturais, orgânicas ou relacionada a patologias específicas.

Aproximadamente $76 \%$ dos pacientes com diagnóstico de depressão apresentavam sintomas somáticos, como uma variedade de sintomas físicos, tensão muscular, tremores corporais, distúrbios de sono, fadiga, tontura, sintomas de ouvido, e sintomas gastrointestinais. Tal situação demonstra a correlação entre sintomas somáticos e a maior duração e gravidade de episódios depressivos, que muitas vezes permanecem como como sintomas residuais, mesmo após o tratamento adequado, resultando em uma diminuição na taxa de remissão da doença e um aumento na taxa de recorrência da depressão (Liu, Y., et al., 2019).

Diante do impacto do TDM na vida coletiva, faz-se necessário compreender quais fatores de risco contribuem para a evolução da depressão maior em uma comunidade acadêmica. Dessa forma, este estudo teve como objetivo realizar análise descritiva do perfil sociodemográfico dos pacientes diagnosticados com transtorno depressivo maior, bem como estabelecer uma correlação a respeito de fatores pessoais, a parâmetros sensibilidade somática, a características fisiológicas a utilização de tratamento farmacológico e entre o grupo com TDM e indivíduos saudáveis.

\section{Metodologia}

Trata-se de estudo observacional transversal retrospectivo, na modalidade descritiva-analítica de caráter quantitativo, que constitui parte do projeto intitulado "Avaliação da sensibilidade e da autoimagem no processo da depressão" em andamento na Universidade Federal do Pará (UFPA), sob aprovação do Comitê de Ética em Pesquisa (CEP) nº 3.236.726. 
A amostra total desta pesquisa foi por conveniência, composta por 50 participantes e os critérios de inclusão foram constituídos por dados de pacientes adultos de ambos os sexos, idade de 18 a 60 anos, avaliados no período de março a maio de 2019.

O grupo transtorno depressivo maior $(\mathrm{GTDM})(\mathrm{n}=25)$ foi composto por pacientes com diagnóstico de transtorno depressivo maior (CID 10: F33), em tratamento farmacológico ou não, que foram atendidos no Serviço de Assistência Psicossocial (SAPS) da UFPA, encaminhados por profissionais de saúde de clínicas psiquiátricas diferentes. O grupo controle (GC) (n=25) foi formado por participantes sem diagnóstico de transtorno depressivo maior, avaliados no Laboratório de Neuroinflamação (LNI) da instituição. Todos os participantes dos dois grupos eram vinculados a UFPA sendo servidores, docentes e discentes da instituição.

Os critérios de exclusão foram idade inferior a 18 anos, diagnósticos psiquiátricos não correspondente com transtorno depressivo maior, uso de antibióticos e de corticoides. Também foram excluídos deste estudo dados de prontuários e banco de dados do projeto integrado que estivessem com escassez ou ausência de informações.

Os critérios descritos acima foram adotados em decorrência dos transtornos psiquiátricos apresentarem diferentes mecanismos fisiopatológicos, bem com a farmacodinâmica desenvolvida pelo uso de antibiótico e corticoides interferirem na manifestação dos sintomas somestésicos, ocasionando baixa fidedignidade dos dados registrados.

Após elegibilidade da amostra foram analisadas fichas de avaliação, elaborada pelos autores, para a coleta de informações referentes ao perfil sociodemográfico, com questões referentes a idade, orientação sexual, estado civil, naturalidade, grau de instrução, massa corporal, estatura, calculado o índice de massa corporal (IMC [por meio da razão entre a massa corporal em $\mathrm{kg}$ e a estatura $\mathrm{em} \mathrm{m}^{2} \mathrm{]}$ ), comorbidades, alergias, hábitos de vida, parâmetros de sensibilidade somática, queda de cabelo, quantidade e qualidade de sono e utilização ou não de medicamentos de pacientes do grupo depressivo e voluntários do grupo controle. O protocolo do estudo foi elaborado de acordo com os critérios STROBE (von Elm, E., et al., 2014).

As informações foram tabuladas no programa Microsoft Office Excel® 2013, para que pudessem ser comparadas e organizadas e, posteriormente, processadas no programa Bioestat versão 5.3.

A avaliação estatística descritiva foi feita nas variáveis sociodemográficas, parâmetros de sensação somática, outras características e na utilização de medicamentos. $\mathrm{O}$ teste do qui-quadrado $\left(\mathrm{x}^{2}\right)$ foi utilizado para aplicar hipótese de associação entre as variáveis definidas com significância de 5\%. O cruzamento descritivo entre os dados do GC e do GTDM foi executado por meio da análise de correlação "Coeficiente de Contingência C" para verificar a ligação entre as variáveis (resultado de $\mathrm{C}=0$, determina que não há associação entre as variáveis, quando $\mathrm{C} \neq 0$, há correlação entre duas variáveis $<0.1$ fraca, 0.1 a 0.3 moderada e $>0.3$ forte; sendo adotado o nível de significância de $5 \%$ - $<<0,05$ ). Os dados obtidos para idade, massa corporal $(\mathrm{Kg})$, estatura $(\mathrm{m}), \mathrm{IMC}\left(\mathrm{Kg} / \mathrm{m}^{2}\right)$ e quantidade de horas dormidas foram avaliados quanto as suas médias e desvios padrão.

\section{Resultados}

Os resultados da Tabela 1 mostram o perfil sociodemográfico dos 50 participantes avaliados. Participaram do estudo discentes, docentes e servidores da instituição, com idade entre 18 e 64 anos, identificou-se que a média de idade aumentou em 11,53\% para o GTDM em relação ao GC, sendo a maioria pertencente ao sexo feminino 29 (58\%). Ao associar as variáveis sociodemográficas com a depressão, verificou-se que houve uma forte associação entre o sexo $(\mathrm{p}=0.0219)$ com esse transtorno mental.

Encontramos uma forte associação estatisticamente significativa consistente ao funcionamento intestinal regular alterado e o TDM, com um crescimento de $100 \%$ ao estabelecer uma comparação entre os grupos. Ainda em relação aos dados de hábitos de vida da comunidade acadêmica, também foi observado um forte coeficiente no teste de contingência nas 
variáveis relacionadas ao grau de instrução, etilismo e tabagismo (Tabela1).

Tabela 1 - Perfil Epidemiológico do GC e GTDM.

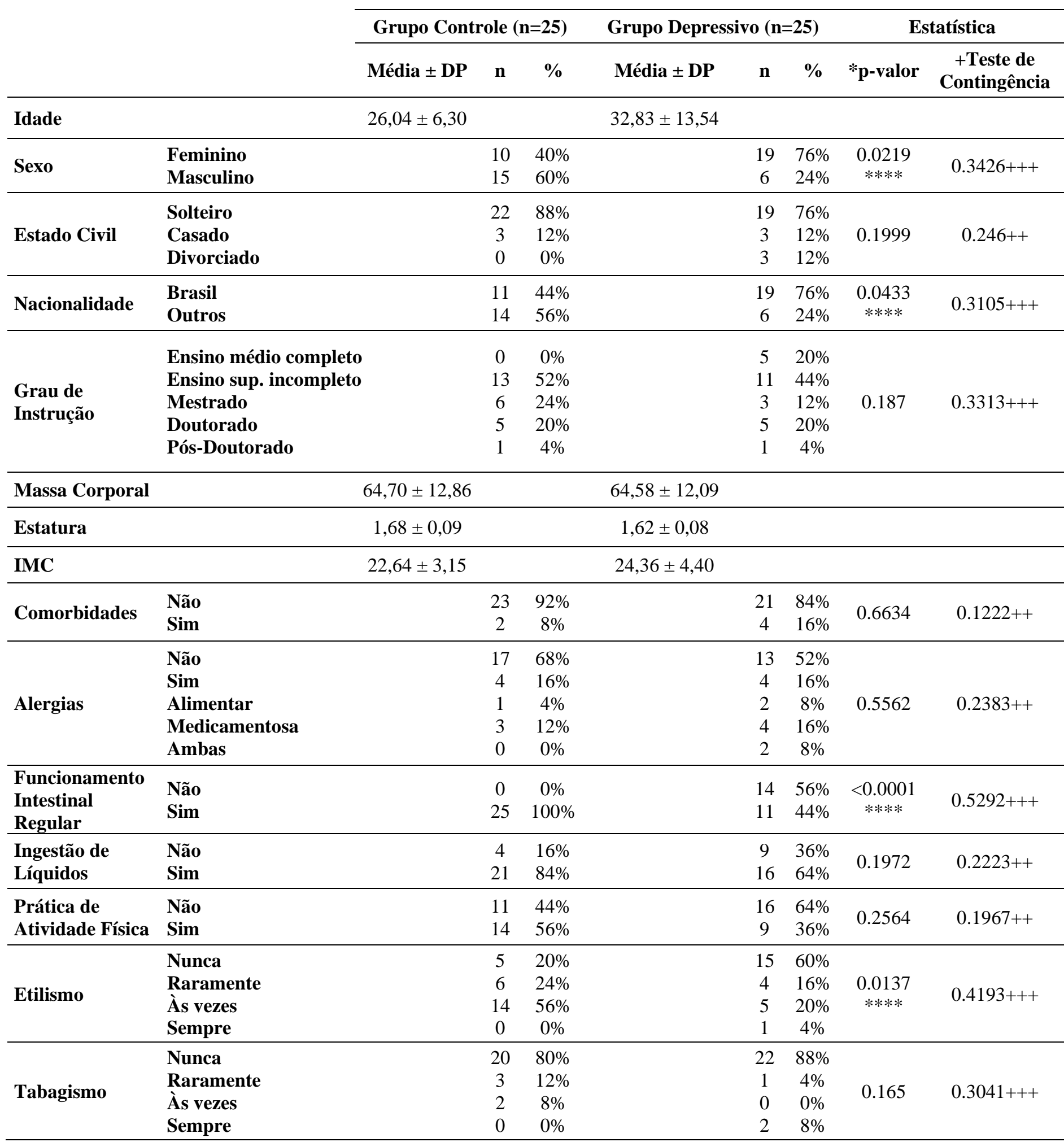

DP: desvio-padrão; n: Frequência; Massa corporal (Kg); Estatura (m); IMC (Kg/m²).

$+\mathrm{C}=0$-Não há associação entre as variáveis; $\mathrm{C} \neq 0$-há correlação entre duas variáveis $(<0.1$ fraca, ++0.1 a 0.3 moderada e $+++>0.3$ forte $)$ $* * * * \mathrm{p}<0,05$. Fonte: Autores. 
Na Tabela 2, dentre as variáveis analisadas referentes as sensações somáticas de temperatura, de percepção de prurido e a ausência de sensibilidade, a análise da sensibilidade térmica para o calor apresentou uma diminuição de 12,5\%, em contrapartida a percepção do frio demonstrou um aumento de 22,2\% em comparação aos dois grupos, mas não houve significância estatística

Quanto ao estudo da percepção do prurido, observou-se que o GTDM obteve uma frequência superior quando comparado ao grupo GC, com um aumento de 69,23\%, dentre esses participantes (n=5), foi observado a presença de prurido extrema. De modo que dentre as variáveis analisadas, a presença do prurido alterado demonstrou associação significativa com o TDM.

Também foi analisada a presença de alterações de sensibilidade em regiões corporais, com um crescimento de $27,27 \%$ entre os grupos. O estudo demonstrou a ausência de sensibilidade em regiões corporais, sendo essas em membros inferiores $(n=5)$, membros superiores $(n=2)$. Embora tenha apresentado aumento de risco não foi estatisticamente significativo.

Tabela 2 - Características de sensação somática a temperatura, prurido e ausência de sensibilidade entre os GC e GTDM.

\begin{tabular}{|c|c|c|c|c|c|c|c|}
\hline & & \multicolumn{2}{|c|}{$\begin{array}{c}\text { Grupo Controle } \\
(n=25)\end{array}$} & \multicolumn{2}{|c|}{$\begin{array}{c}\text { Grupo Depressivo } \\
(\mathrm{n}=25)\end{array}$} & \multicolumn{2}{|c|}{ Estatística } \\
\hline & & $\mathbf{n}$ & $\%$ & $\mathbf{n}$ & $\%$ & $*$ p-valor & $\begin{array}{c}\text { +Teste de } \\
\text { Contingência } \\
\end{array}$ \\
\hline \multirow{2}{*}{$\begin{array}{l}\text { Sensibilidade } \\
\text { Térmica }\end{array}$} & Calor & 18 & $72 \%$ & 14 & $56 \%$ & \multirow[b]{2}{*}{0.3768} & \multirow[b]{2}{*}{$0.1644++$} \\
\hline & Frio & 7 & $28 \%$ & 11 & $44 \%$ & & \\
\hline \multirow{2}{*}{ Prurido } & Não & 23 & $92 \%$ & 14 & $56 \%$ & \multirow{2}{*}{$0.0099 * * * *$} & \multirow{2}{*}{$0.3796+++$} \\
\hline & Sim & 2 & $8 \%$ & 11 & $44 \%$ & & \\
\hline \multirow{2}{*}{$\begin{array}{l}\text { Ausência de } \\
\text { Sensibilidade }\end{array}$} & Não & 21 & $84 \%$ & 18 & $72 \%$ & \multirow{2}{*}{0.4947} & \multirow{2}{*}{$0.1433++$} \\
\hline & Sim & 4 & $16 \%$ & 7 & $28 \%$ & & \\
\hline
\end{tabular}

n: Frequência. $+\mathrm{C}=0$-Não há associação entre as variáveis; $\mathrm{C} \neq 0$-há correlação entre duas variáveis $(<0.1$ fraca, ++0.1 a 0.3 moderada e $+++>0.3$ forte) $* * * * p<0,05$. Fonte: Autores.

Ao verificar outras características entre os dois grupos na Tabela 3, houve associação estatisticamente significante entre a queda de cabelo e a qualidade do sono no grupo com transtorno depressivo. Foi identificado que houve um aumento de $40 \%$ entre os grupos analisados.

A qualidade do sono foi a percepção mais significativa e de maior diferença entre os grupos comparados. Observou-se um aumento de $100 \%$ entre os grupos.

Além disso, a ponderação no que concerne quantidade de horas dormidas mostrou que no GC, nota-se somente um padrão de horas dormidas sem o uso de medicamentos, com uma média de 6,76 horas de sono. Ao equiparar com o GTDM que apresentou uma média de 4,31 horas dormidas sem medicamento, destacando ainda, uma diminuição de $22,13 \%$ nesse padrão quantitativo de sono. 
Tabela 3 - Outras características qualitativas e quantitativas do GC e GTDM.

\begin{tabular}{|c|c|c|c|c|c|c|c|}
\hline & & \multicolumn{2}{|c|}{$\begin{array}{c}\text { Grupo Controle } \\
(\mathrm{n}=25)\end{array}$} & \multicolumn{2}{|c|}{$\begin{array}{c}\text { Grupo Depressivo } \\
(n=25)\end{array}$} & \multicolumn{2}{|c|}{ Estatística } \\
\hline & & $\mathbf{n}$ & $\%$ & $\mathbf{n}$ & $\%$ & *p-valor & $\begin{array}{c}\text { Teste de } \\
\text { Contingência } \\
\end{array}$ \\
\hline Queda de & Não & 16 & $64 \%$ & 4 & $16 \%$ & \multirow{2}{*}{$0.0015 * * * *$} & \multirow{2}{*}{$0.4399+++$} \\
\hline Cabelo & Sim & 9 & $36 \%$ & 21 & $84 \%$ & & \\
\hline Qualidade do & Insônia & 0 & $0 \%$ & 24 & $96 \%$ & \multirow{2}{*}{$<0.0001 * * * *$} & \multirow{2}{*}{$0.6928+++$} \\
\hline Sono & Normal & 25 & $100 \%$ & 1 & $4 \%$ & & \\
\hline \multirow{2}{*}{$\begin{array}{l}\text { Quantidade } \\
\text { de horas } \\
\text { dormidas }\end{array}$} & Sem medicamento & \multicolumn{2}{|c|}{$6,76 \pm 1,69$} & \multicolumn{2}{|c|}{$4,31 \pm 1,72$} & & \\
\hline & Medicamentosa & \multicolumn{2}{|c|}{ Não informado } & \multicolumn{2}{|c|}{$7,12 \pm 1,24$} & & \\
\hline
\end{tabular}

n: Frequência. $+\mathrm{C}=0$-Não há associação entre as variáveis; $\mathrm{C} \neq 0$-há correlação entre duas variáveis $(<0.1$ fraca, ++0.1 a 0.3 moderada e $+++>0.3$ forte) $* * * * p<0,05$. Fonte: Autores.

A análise comparativa entre o uso ou não de medicamentos pelos dois grupos, na Tabela 4, gerou um valor de $\mathrm{p}$ estatisticamente significativo de $\mathrm{p}<0.0001$, com um indicativo de $100 \%$ de aumento percentual entre os grupos.

Dentre esses medicamentos consumidos, a classe terapêutica exposta na Tabela 5, a mais utilizada foi de antidepressivos 10 (52,63\%), seguido por antipsicóticos 5 (26,32\%), benzodiazepínicos 3 (15,79\%) e hipnótico 1 (5,26\%). A respeito de pacientes que faziam uso de terapia combinada, os resultados mostram que 10 (40\%) utilizavam apenas uma medicação, 8 (32\%), utilizavam duas medicações e 3 (12\%) associavam três intervenções farmacológicas em comparação ao GC $25(100 \%)$ que não fazia uso de medicamentos.

Tabela 4 - Utilização de medicamentos entre o GC e GTDM.

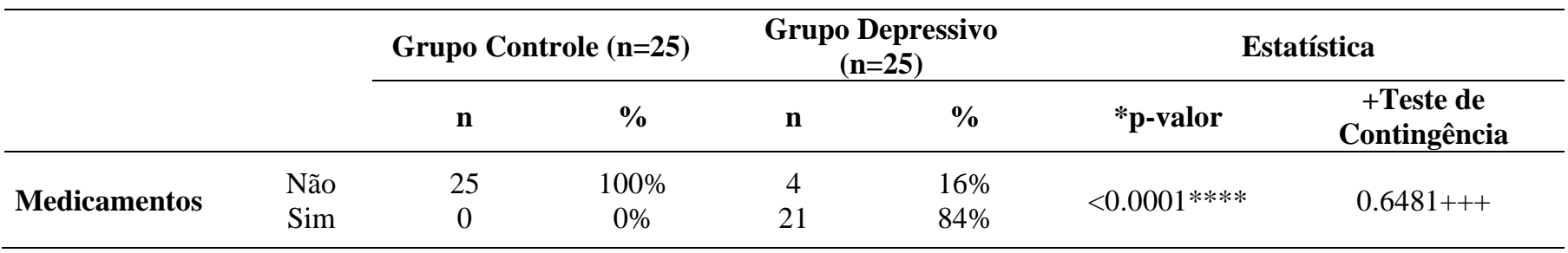

n: Frequência. $+C=0$-Não há associação entre as variáveis; $C \neq 0$-há correlação entre duas variáveis $(<0.1$ fraca, ++0.1 a 0.3 moderada e $+++>0.3$ forte) $* * * * p<0,05$. Fonte: Autores.

Tabela 5 - Classe Terapêutica e Interação Medicamentosa analisada no GTDM.

\begin{tabular}{llcc} 
& & Frequência & $\%$ \\
\cline { 3 - 4 } Classe Terapêutica $(\mathbf{n = 1 9 )}$ & Antidepressivos & 10 & $52.63 \%$ \\
& Benzodiazepínicos & 3 & $15.79 \%$ \\
& Antipsicóticos & 5 & $26.32 \%$ \\
& Hipnóticos & 1 & $5.26 \%$ \\
\hline \multirow{2}{*}{ Interação Medicamentosa $(\mathbf{n = 2 5})$} & Não Usam & 4 & $16 \%$ \\
& Usam apenas 1 & 10 & $40 \%$ \\
& Usam apenas 2 & 8 & $32 \%$ \\
& Usam apenas 3 & 3 & $12 \%$ \\
\hline
\end{tabular}

Fonte: Autores. 
Não encontramos uma associação entre sintomas depressivos e as seguintes variáveis: idade, massa corporal, estatura, IMC, estado civil, comorbidades, alergias, ingestão de líquidos, prática de atividade física, sensibilidade térmica e ausência de sensibilidade (Tabelas 1 e 2).

\section{Discussão}

A depressão é um distúrbio heterogêneo e multifatorial, caracterizado por uma série de sintomas cognitivos, afetivos e somáticos com uma prevalência muito alta em todo o mundo (Athira, K. et al., 2020). A etiologia do transtorno depressivo maior (TDM) é complexa, com componentes psicológicos, biológicos e ambientais que cursam com estados de cronicidade e de remissão durante a patogênese da doença, afetando a qualidade de vida e a funcionalidade do indivíduo (Otte C, et al., 2016; Athira, K. et al., 2020).

Em decorrência de uma ampla variedade de tipos de transtorno depressivo e as suas interações com as diferentes faixas etárias, seus valores apresentam um grande intervalo de variação semelhantes às deste estudo. Sabe-se que a maioria dos casos de problemas e desordens mentais em adultos começam com manifestações durante a adolescência e o começo da idade adulta tendo uma faixa dos 12 até 25 anos (Wu, W., et al., 2020). Em ambos os sexos, a média de idade relatada de início do TDM é de aproximadamente 25 anos e o período de pico de risco para o início de TDM varia desde metade ao final da adolescência até o início dos 40 anos (Bromet, E., et al., 2011)

Os resultados mostram a prevalência de TDM entre as mulheres 19 (76\%) em comparação com os homens 6 (24\%), um achado que tem sido relatado de maneira consistente em estudos de epidemiologia psiquiátrica (Salk, R. H., et al., 2017). Existe uma correlação entre fatores sociais, comportamentais, psicológicos e biológicos que possivelmente interagem uns com os outros e favorecem o risco de mulheres em desenvolver o transtorno depressivo.

Dentre eles a suscetibilidade biológica associada ao mecanismo hormonal, na qual as mulheres têm um risco duas vezes maior de desenvolver TDM do que os homens após a puberdade, a desigualdade social favorecendo a facilidade de mulheres a buscar ajuda quando necessário e as influências comportamentais que inibem os homens de demonstrações de tristeza (Salk, R. H., et al., 2017). Alguns estudos também demonstram diferenças na expressão de transportadores de serotonina associados à depressão em homens e mulheres (Chang CC., et al., 2016).

Dos transtornos mentais mais frequentes em estudantes universitários a depressão maior é particularmente prevalente entre as mulheres. Um estudo transversal com objetivo de determinar os níveis de depressão, ansiedade e estresse e a prevalência de depressão e fatores associados, em mulheres universitárias espanholas revelam uma porcentagem alarmante de universitárias que experimentam depressão e estresse severo / muito severo (Blanco, V., et al., 2021).

Um outro aspecto social identificado neste estudo foi a presença do grau de instrução entre a comunidade acadêmica que apresentaram ou não depressão, revelando uma associação entre o maior nível de escolaridade e o TDM. Sobre isso, é importante destacar a presença de uma problemática de saúde mental entre a população estudada, evidências encontradas na literatura sugerem que o menor nível de escolaridade está vinculado a um maior risco de sintomas depressivos (Cohen, A. K., 2020).

Uma pesquisa recente avaliou a relação entre desempenho educacional e depressão e obtiveram como resultado que o maior desempenho no âmbito estudantil está associado a um risco reduzido de depressão na meia-idade em uma amostra nacionalmente representativa de adultos nos Estados Unidos. (Mirowsky J. 2017)

A pesquisa dos hábitos de vida demonstrou uma correlação existente entre o funcionamento intestinal regular e o TDM. Distúrbios no equilíbrio do eixo intestino-microbiota têm sido associados à fisiopatologia da depressão, uma vez que o eixo intestino-cérebro envolve uma sinalização bioquímica entre o trato gastrointestinal (GI) e o sistema nervoso central (SNC). De modo que quando a estrutura GI está afetada, a funcionalidade da barreira protetora fica comprometida, levando a 
um aumento da permeabilidade intestinal e à penetração de substâncias que podem alterar as funções fisiológicas e ativar a resposta imune, que resulta em níveis de mediadores da inflamação que desencadeiam doenças do espectro neuropsiquiátrico (Generoso, J. S., et al., 2021).

De acordo com ensaio um clínico duplo-cego para comparar pacientes com transtorno depressivo maior e a utilização de probióticos, 110 pacientes deprimidos foram randomizados para receber probióticos( B. longum e L. helveticus ), prebióticos (galacto-oligossacarídeo) ou placebo por 8 semanas, obtendo como resultado após o tratamento, efeitos potencialmente benéficos na melhoria dos sintomas de transtornos depressivos, com diminuição significativa na pontuação do Inventário de Depressão de Beck, embora a suplementação de prebióticos não tenha tido efeito. (Kazemi A., et al., 2019)

Uma análise da literatura nos mostra uma relação entre TDM e consumo excessivo de álcool entre adultos jovens. Um estudo com estudantes universitários descobriu que os sintomas depressivos foram associados ao consumo excessivo de álcool, uso problemático de álcool e maior uso diário de álcool (Pedrelli, P., et al., 2016). Contudo, de acordo com a pesquisa, a variável etilismo apresentou uma correlação positiva entre os grupos, sendo um achado importante que aponta um aspecto de autocuidado entre os grupos, principalmente entre o GTDM, uma vez que para pacientes deprimidos, o uso do álcool é um obstáculo ao sucesso do tratamento ao atuar como um depressor do sistema nervoso central (SNC) que exacerba os sintomas depressivos (Kane, C. J., \& Drew, P. D. 2016).

Neste estudo, os resultados mostraram que o uso de nicotina entre os grupos é baixo; todavia, o grupo TDM apresentou um percentual de indivíduos fumantes 2 (8\%) que corroboram com os dados encontrados na literatura. Estudos indicam a existência de uma correlação entre o uso de tabaco e o surgimento de doenças mentais, dados epidemiológicos recentes retratam que a taxa de uso de tabaco em indivíduos clinicamente deprimidos é cerca de duas vezes a taxa na população em geral. Tal situação é vinculada a hipótese da automedicação provocada pela nicotina, na qual as pessoas recorrem ao tabagismo para aliviar seus sintomas e, portanto, sugere que os sintomas de depressão e ansiedade podem levar ao tabagismo (Mathew, A. R., et al., 2017).

Ademais, indivíduos com transtorno depressivo são mais suscetíveis a fumar, tendem a começar a fumar mais cedo, fumar mais cigarros por dia e são menos propensos a parar de fumar com sucesso do que indivíduos sem depressão (Mathew, A. R., et al., 2017).

Dados de uma revisão sistemática e meta-análise estabeleceram uma associação entre clima quente e resultados ruins de saúde mental, corroborando com nossos achados de fatores de risco associados a perpetuação dos sintomas depressivos, os resultados mostram que um aumento de $1^{\circ} \mathrm{C}$ na temperatura foi associado a um aumento significativo na morbidade, como transtornos de humor, transtornos mentais orgânicos, esquizofrenia, distúrbios neuróticos e de ansiedade; assim como sugerem uma maior vulnerabilidade mental para as populações que vivem em zonas de clima tropical e subtropical (Liu J., et al., 2021).

Chamou a atenção que nas duas variações de temperatura estabelecidas, houve uma frequência de 4 indivíduos indicando calor extremo e 4 indivíduos relatando frio extremo, todos do GTDM, apesar de não existir uma associação significativa.

No presente estudo, observou-se que a percepção do prurido alterada é um fator associado ao desenvolvimento do TDM. O prurido é um sintoma comumente relatado em pacientes psiquiátricos e, da mesma forma, comorbidades psiquiátricas, incluindo ansiedade e depressão, são frequentemente vistas em pacientes com prurido crônico (Lee HG., et al., 2017). De acordo com um estudo transversal que analisou associações entre prurido crônico e condições psicológicas, incluindo insônia e depressão, os pacientes com sintomas de insônia ou depressão tinham prurido significativamente mais intenso do que pacientes sem sintomas psicológicos (Lee, J., et al., 2021). 
O estresse psicológico e os traços de personalidade influenciam de forma forte na sensação da coceira, assim como estes fatores psicológicos desenvolvem um papel importante no desenvolvimento, modificação e manutenção da coceira (Jafferany, M., \& Davari, M. E. 2018).

Verificou-se também presença de alterações de sensibilidade em regiões corporais, no qual a investigação indicou que pacientes com TDM tem como sintomatologia a percepção da sensibilidade ausente em uma parte do corpo. O impacto da depressão no limiar absoluto da percepção sensorial indica que os indivíduos deprimidos apresentaram limiares de percepção sensorial mais elevados, ou seja, são menos sensíveis aos estímulos em geral em comparação com os indivíduos não deprimidos. (Meerwijk E., et al., 2013).

$\mathrm{Na}$ interpretação de outras características identificadas neste estudo, a relação entre a queda de cabelo foi maior observada em indivíduos com TDM em relação aos controles saudáveis. A queda de cabelo, principalmente no couro cabeludo, afeta negativamente a aparência estética e a autoestima dos indivíduos. Portanto, os pacientes que sofrem de alopecia areata (AA), uma doença cutânea autoimune, podem estar sujeitos a várias doenças psiquiátricas e a maiores riscos de desenvolver transtorno depressivo maior (TDM) (Cakirca, G., et al., 2020).

Um estudo recente propôs uma avaliação de uma associação bidirecional entre transtorno depressivo maior e AA, que incluiu pacientes de 10 a 90 anos de idade e confirmou que pacientes com AA correm o risco de desenvolver TDM, assim como, ter TDM também parece ser um fator de risco significativo para o desenvolvimento de AA (Vallerand, I. A., et al., 2019).

Ademais, a queda de cabelo pode ser um efeito colateral do uso de medicamentos antidepressivos, de acordo com o estudo sobre queda de cabelo associada ao escitalopram e a ação dos inibidores de recaptação de serotonina (ISRS ou SSRI), esses podem causar efeitos danosos relacionados ao cabelo e à pele, acredita-se que os SSRI podem afetar a fase telógena da produção do cabelo, mudando sua estrutura. (Karabulut, İ. Y., et al., 2021)

Este trabalho identificou que de todas as variáveis avaliadas, a qualidade do sono foi a percepção mais significativa e de maior diferença entre os grupos comparados, demonstrando um alto potencial da insônia como um fator de risco para o TDM. De acordo com uma meta-análise de estudos de coorte prospectivos sobre insônia e o risco de depressão, após analisar trinta e quatro estudos de coorte envolvendo 172.077 participantes com um período de acompanhamento médio de 60,4 meses. A análise estatística sugeriu uma relação positiva entre insônia e um risco aumentado de depressão (Li L., et al., 2016).

A relação entre insônia e sintomas de humor é bidirecional, pois o sono insatisfatório pode preceder um episódio de transtorno depressivo maior e o humor deprimido pode interromper os padrões normais de sono. Além disso, é 3 vezes mais provável que o transtorno depressivo maior se desenvolva em indivíduos com insônia do que naqueles sem (Murphy, M. J., \& Peterson, M. J. 2015).

Distúrbios do sono estão associados a mecanismos vinculados ao TDM, a hipótese da inflamação sugere que em pacientes deprimidos ocorre um aumento nos níveis séricos citocinas inflamatórias (por exemplo, IL-6 e TNF) ao longo do dia em relação a indivíduos não deprimidos. expressão do gene inflamatório. Ademais, a teoria que preconiza alterações nas vias bioquímicas nos níveis de monoaminas está associada a fisiopatologia da depressão (Fang, H., et al., 2019)

O uso de medicamentos se deu de maneira superior no GTDM em equiparação ao GC, mostrando, assim, a presença de uma intervenção farmacológica associada a sintomatologia depressiva. Vários agentes farmacológicos foram desenvolvidos para tratar a depressão, incluindo várias classes de medicamentos antidepressivos, cada um definido por um conjunto distinto de características clínicas.

Como terapia de primeira linha, ao tratar pessoas com depressão maior, as diretrizes atuais recomendam a monoterapia com antidepressivos como tratamento farmacológico de inicial (American Psychiatric Association, 2014), a fim de estabelecer uma dosagem ideal de um único agente para tratar as queixas de humor e de sono. A maioria dos medicamentos 
antidepressivos tem algum efeito na regulação do sono, seja sedativo ou ativador; portanto, o tratamento eficaz dos sintomas de humor pode agravar os distúrbios do sono existentes ou provocar novos problemas de sono.

Muitos pacientes apresentam sintomas que não podem ser controlados adequadamente por um único agente, à medida que que pacientes com TDM não respondem satisfatoriamente à monoterapia com antidepressivo de primeira linha, estratégias adicionais de tratamento com outros compostos psiquiátricos são frequentemente estabelecidas (Ogawa, Y., et al., 2019).

Os benzodiazepínicos compõem uma classe de drogas psicotrópicas usados principalmente para pessoas com sintomas de ansiedade (ansiolíticos, tranquilizantes) e como medicamentos hipnóticos para pessoas com insônia; O possível benefício dos antipsicóticos nos sintomas somáticos pode ser devido aos seus efeitos analgésicos (Ogawa, Y, et al., 2019). Outrossim, diretrizes estabelecem um valor limitado de benzodiazepínicos como terapia combinada e permitem que os benzodiazepínicos possam ser usados por um curto período com um antidepressivo se as pessoas apresentarem sintomas de ansiedade ou insônia (American Psychiatric Association, 2014).

Como limitação do estudo, por se tratar de respostas subjetivas dadas pelos entrevistados, a análise de variáveis quantitativas como medidas corporais, massa $(\mathrm{Kg})$ e estatura $(\mathrm{m})$ para determinar o $\mathrm{IMC}\left(\mathrm{Kg} / \mathrm{m}^{2}\right)$, não foi fidedigna às medidas reais. Outra limitação refere-se ao fato de ser uma ficha de avaliação com muitas perguntas, podendo ser cansativo para os participantes.

\section{Conclusão}

Com base nos resultados obtidos conclui-se que os pacientes com transtorno depressivo maior apresentam fatores de risco próprios do indivíduo e sintomas somáticos que influenciam no curso de resolução da doença. O sexo, o grau de instrução, o funcionamento intestinal regular e os hábitos de vida como o etilismo e o tabagismo parecem ser características sociodemográficas intervenientes na probabilidade de ocorrência do transtorno depressivo maior no meio acadêmico. Além disso, o estudo mostrou que as alterações ocasionadas pela patogênese da depressão podem repercutir de maneira sistêmica e provocar sintomas somáticos como a presença de prurido, a queda de cabelo e a qualidade do sono que afetam o bem-estar do indivíduo.

Sugere-se a realização de mais estudos que relacionem características sociodemográficas, a presença de sintomas somáticos e interação medicamentosa para, assim, identificar possíveis fatores de risco associados ao quadro de depressão maior na população universitária, a fim de que estas condições contribuam para um diagnóstico precoce, uma estratégia de tratamento interdisciplinar e multidisciplinar que reúna as particularidades da doença e, assim, promovam uma harmonização entre os cuidados e a evolução do curso do TDM para um prognóstico resolutivo e integral.

\section{Referências}

American Psychiatric Association. (2014). Manual diagnóstico e estatístico de transtornos mentais: DSM-5. 5. ed.

Athira, K. V., Bandopadhyay, S., Samudrala, P. K., Naidu, V., Lahkar, M., \& Chakravarty, S. (2020). An Overview of the Heterogeneity of Major Depressive Disorder: Current Knowledge and Future Prospective. Current neuropharmacology, 18(3), 168-187. https://doi.org/10.2174/1570159X17666191001142934

Beurel, E., Toups, M., \& Nemeroff, C. B. (2020). The Bidirectional Relationship of Depression and Inflammation: Double Trouble. Neuron, 107(2), 234-256. https://doi.org/10.1016/j.neuron.2020.06.002

Birur, B., Amrock, E. M., Shelton, R. C., \& Li, L. (2017). Sex Differences in the Peripheral Immune System in Patients with Depression. Frontiers in psychiatry, 8, 108. https://doi.org/10.3389/fpsyt.2017.00108

Blanco, V., Salmerón, M., Otero, P., \& Vázquez, F. L. (2021). Symptoms of Depression, Anxiety, and Stress and Prevalence of Major Depression and Its Predictors in Female University Students. International journal of environmental research and public health, 18(11), 5845. https://doi.org/10.3390/ijerph18115845

Bromet, E., Andrade, L. H., Hwang, I., Sampson, N. A., Alonso, J., de Girolamo, G., de Graaf, R., Demyttenaere, K., Hu, C., Iwata, N., Karam, A. N., Kaur, J., Kostyuchenko, S., Lépine, J. P., Levinson, D., Matschinger, H., Mora, M. E., Browne, M. O., Posada-Villa, J., Viana, M. C., ... Kessler, R. C. (2011). Cross-national epidemiology of DSM-IV major depressive episode. BMC medicine, 9, 90. https://doi.org/10.1186/1741-7015-9-90 
Cakirca, G., Manav, V., Celik, H., Saracoglu, G., \& Yetkin, E. N. (2020). Effects of anxiety and depression symptoms on oxidative stress in patients with alopecia areata. Postepy dermatologii i alergologii, 37(3), 412-416. https://doi.org/10.5114/ada.2019.83879

Chang, C. C., Chang, H. A., Fang, W. H., Chang, T. C., \& Huang, S. Y. (2017). Gender-specific association between serotonin transporter polymorphisms (5HTTLPR and rs25531) and neuroticism, anxiety and depression in well-defined healthy Han Chinese. Journal of affective disorders, 207, 422-428. https://doi.org/10.1016/j.jad.2016.08.055

Cohen, A. K., Nussbaum, J., Weintraub, M., Nichols, C. R., \& Yen, I. H. (2020). Association of Adult Depression With Educational Attainment, Aspirations, and Expectations. Preventing chronic disease, 17, E94. https://doi.org/10.5888/pcd17.200098

Fang, H., Tu, S., Sheng, J., \& Shao, A. (2019). Depression in sleep disturbance: A review on a bidirectional relationship, mechanisms and treatment. Journal of cellular and molecular medicine, 23(4), 2324-2332. https://doi.org/10.1111/jcmm.14170

Fernández-Rodríguez, C., Soto-López, T., \& Cuesta, M. (2019). Needs and demands for psychological care in university students. Psicothema, 31(4), 414421. https://doi.org/10.7334/psicothema2019.78

Generoso, J. S., Giridharan, V. V., Lee, J., Macedo, D., \& Barichello, T. (2021). The role of the microbiota-gut-brain axis in neuropsychiatric disorders. Revista brasileira de psiquiatria (Sao Paulo, Brazil : 1999), 43(3), 293-305. https://doi.org/10.1590/1516-4446-2020-0987

Gonçalves, A. M. C., Teixeira, M. T. B., Gama, J. R.A., Lopes, C. S., Silva, G. A., Gamarra, C. J. et al. (2017). Prevalência de depressão e fatores associados em mulheres atendidas pela Estratégia de Saúde da Família. J BrasPsquiatr., 67(2), 101-109. doi: 10.1590/0047-2085000000192

Jafferany, M., \& Davari, M. E. (2019). Itch and psyche: psychiatric aspects of pruritus. International journal of dermatology, 58(1), 3-23. https://doi.org/10.1111/ijd.14081

Karabulut, İ. Y., Gokcay, H., \& Belli, H. (2021). Hair Loss Associated with Escitalopram: Do SSRIs Affect Melatonin at the Hair Follicle?. Psychiatria Danubina, 33(2), 187-188. https://doi.org/10.24869/psyd.2021.187

Kane, C. J., \& Drew, P. D. (2016). Inflammatory responses to alcohol in the CNS: nuclear receptors as potential therapeutics for alcohol-induced neuropathologies. Journal of leukocyte biology, 100(5), 951-959. https://doi.org/10.1189/jlb.3MR0416-171R

Kazemi, A., Noorbala, A. A., Azam, K., Eskandari, M. H., \& Djafarian, K. (2019). Effect of probiotic and prebiotic vs placebo on psychological outcomes in patients with major depressive disorder: A randomized clinical trial. Clinical nutrition (Edinburgh, Scotland), 38(2), 522-528. https://doi.org/10.1016/j.clnu.2018.04.010

Lee, H. G., Stull, C., \& Yosipovitch, G. (2017). Psychiatric disorders and pruritus. Clinics in dermatology, 35(3), 273-280. https://doi.org/10.1016/j.clindermatol.2017.01.008

Lee, J., Suh, H., Jung, H., Park, M., \& Ahn, J. (2021). Association between chronic pruritus, depression, and insomnia: A cross-sectional study. JAAD international, 3, 54-60. https://doi.org/10.1016/j.jdin.2021.02.004

Li, L., Wu, C., Gan, Y., Qu, X., \& Lu, Z. (2016). Insomnia and the risk of depression: a meta-analysis of prospective cohort studies. BMC psychiatry, 16(1), 375. https://doi.org/10.1186/s12888-016-1075-3

Liu, J., Varghese, B. M., Hansen, A., Xiang, J., Zhang, Y., Dear, K., Gourley, M., Driscoll, T., Morgan, G., Capon, A., \& Bi, P. (2021). Is there an association between hot weather and poor mental health outcomes? A systematic review and meta-analysis. Environment international, 153 , 106533. https://doi.org/10.1016/j.envint.2021.106533

Liu, Y., Zhao, J., Fan, X., \& Guo, W. (2019). Dysfunction in Serotonergic and Noradrenergic Systems and Somatic Symptoms in Psychiatric Disorders. Frontiers in psychiatry, 10, 286. https://doi.org/10.3389/fpsyt.2019.00286

Mathew, A. R., Hogarth, L., Leventhal, A. M., Cook, J. W., \& Hitsman, B. (2017). Cigarette smoking and depression comorbidity: systematic review and proposed theoretical model. Addiction (Abingdon, England), 112(3), 401-412. https://doi.org/10.1111/add.13604

Meerwijk, E. L., Ford, J. M., \& Weiss, S. J. (2013). Brain regions associated with psychological pain: implications for a neural network and its relationship to physical pain. Brain imaging and behavior, 7(1), 1-14. https://doi.org/10.1007/s11682-012-9179-y

Medeiros, S. P., Oliveira, A. M. N. de, Silva, M. R. S. da, Freitag, V. L., Afonso, M. dos S., \& Brum, A. N. (2020). Práticas integrativas e complementares: estratégia de cuidado por meio do Reiki em pessoas com depressão. Research, Society and Development, 9(2), e127922149. https://doi.org/10.33448/rsdv9i2.2149

Mirowsky, J., \& Ross, C.E. (2003). Education, Social Status, and Health (1st ed.). Routledge. https://doi.org/10.4324/9781351328081

Murphy, M. J., \& Peterson, M. J. (2015). Sleep Disturbances in Depression. Sleep medicine clinics, 10(1), 17-23. https://doi.org/10.1016/j.jsmc.2014.11.009 $\begin{array}{lllll}\text { Organização Mundial da } & \text { da } & \text { (2018). } & \text { Folha }\end{array}$ https://www.paho.org/bra/index.php?option=com_content \&view=article\&id=5635:folha-informativa-depressao\&Itemid=1095

Ogawa, Y., Takeshima, N., Hayasaka, Y., Tajika, A., Watanabe, N., Streiner, D., \& Furukawa, T. A. (2019). Antidepressants plus benzodiazepines for adults with major depression. The Cochrane database of systematic reviews, 6(6), CD001026. https://doi.org/10.1002/14651858.CD001026.pub2

Otte, C., Gold, S. M., Penninx, B. W., Pariante, C. M., Etkin, A., Fava, M., Mohr, D. C., \& Schatzberg, A. F. (2016). Major depressive disorder. Nature reviews. Disease primers, 2, 16065. https://doi.org/10.1038/nrdp.2016.65

Pedrelli, P., Shapero, B., Archibald, A., \& Dale, C. (2016). Alcohol use and depression during adolescence and young adulthood: a summary and interpretation of mixed findings. Current addiction reports, 3(1), 91-97. https://doi.org/10.1007/s40429-016-0084-0 
Research, Society and Development, v. 10, n. 16, e320101623751, 2021

(CC BY 4.0) | ISSN 2525-3409 | DOI: http://dx.doi.org/10.33448/rsd-v10i16.23751

Pitsillou, E., Bresnehan, S. M., Kagarakis, E. A., Wijoyo, S. J., Liang, J., Hung, A., \& Karagiannis, T. C. (2020). The cellular and molecular basis of major depressive disorder: towards a unified model for understanding clinical depression. Molecular biology reports, 47(1), 753-770. https://doi.org/10.1007/s11033-019-05129-3

Prado, CEP. (2016). Occupational stress: causes and consequences. Rev Bras Med Trab, 14(3):285-289

Salk, R. H., Hyde, J. S., \& Abramson, L. Y. (2017). Gender differences in depression in representative national samples: Meta-analyses of diagnoses and symptoms. Psychological bulletin, 143(8), 783-822. https://doi.org/10.1037/bul0000102

Santos, K., Ferreira, V., da Silva, P., \& Vilella, D. (2018). Aplicabilidade do inventário de Beck nos acadêmicos de enfermagem em uma instituição do sul de minas gerais. Enfermagem em Foco, 9(3). doi:https://doi.org/10.21675/2357-707X.2018.v9.n3.1214

Silva, Érika Correia, \& Tucci, Adriana Marcassa. (2018). Correlação entre ansiedade e consumo de álcool em estudantes universitários. Psicologia: teoria e prática, 20(2), 107-119. https://dx.doi.org/10.5935/1980-6906/psicologia.v20n2p107-119

Stetler, C., \& Miller, G. E. (2011). Depression and hypothalamic-pituitary-adrenal activation: a quantitative summary of four decades of research. Psychosomatic medicine, 73(2), 114-126. https://doi.org/10.1097/PSY.0b013e31820ad12b

Vallerand, I. A., Lewinson, R. T., Parsons, L. M., Hardin, J., Haber, R. M., Lowerison, M. W., Barnabe, C., \& Patten, S. B. (2019). Assessment of a Bidirectional Association Between Major Depressive Disorder and Alopecia Areata. JAMA dermatology, 155(4), 475-479. https://doi.org/10.1001/jamadermatol.2018.4398

von Elm, E., Altman, D. G., Egger, M., Pocock, S. J., Gøtzsche, P. C., Vandenbroucke, J. P., \& STROBE Initiative (2014). The Strengthening the Reporting of Observational Studies in Epidemiology (STROBE) Statement: guidelines for reporting observational studies. International journal of surgery (London, England), 12(12), 1495-1499. https://doi.org/10.1016/j.ijsu.2014.07.013

World Health Organization. (WHO). (2018). Depression and other common mental disorders: global health estimates. Available from: https://apps.who.int/iris/handle/10665/254610.

Wu, W., Zhang, Y., Jiang, J., Lucas, M. V., Fonzo, G. A., Rolle, C. E., Cooper, C., Chin-Fatt, C., Krepel, N., Cornelssen, C. A., Wright, R., Toll, R. T., Trivedi, H. M., Monuszko, K., Caudle, T. L., Sarhadi, K., Jha, M. K., Trombello, J. M., Deckersbach, T., Adams, P., ... Etkin, A. (2020). An electroencephalographic signature predicts antidepressant response in major depression. Nature biotechnology, 38(4), 439-447. https://doi.org/10.1038/s41587-019-0397-3 